\title{
Horizons/Théâtre
}

Revue d'études théâtrales

\section{Retour sur la programmation du focus Afrique subsaharienne du festival d'Avignon 2017}

\section{Pierre Katuszewski}

\section{(2) OpenEdition}

1 Journals

\section{Édition électronique}

URL : https://journals.openedition.org/ht/1256

DOI : 10.4000/ht. 1256

ISSN : 2678-5420

\section{Éditeur}

Presses universitaires de Bordeaux

\section{Édition imprimée}

Date de publication : 31 décembre 2018

Pagination : 136-149

ISBN : 979-10-300-0318-5

ISSN : 2261-4591

\section{Référence électronique}

Pierre Katuszewski, « Retour sur la programmation du focus Afrique subsaharienne du festival d'Avignon 2017 », Horizons/Théâtre [En ligne], 13 | 2018, mis en ligne le 01 janvier 2019, consulté le 17 mai 2022. URL : http://journals.openedition.org/ht/1256 ; DOI : https://doi.org/10.4000/ht.1256

\section{(c) $(1) \odot$}

La revue Horizons/Théâtre est mise à disposition selon les termes de la Licence Creative Commons Attribution - Pas d'Utilisation Commerciale - Pas de Modification 4.0 International. 


\section{PieRRe KatuszeWSKI}

Pierre Katuszewski est maître de conférences en Arts du spectacle à l'Université Bordeaux Montaigne et membre de l'EA CLARE/ARTES. Il est l'auteur de Le Théâtre de Pier Paolo Pasolini (Ides et Calendes, 2015) et de Ceci n'est pas un fantôme. Essai sur les personnages de fantômes dans les théâtres antique et contemporain (Kimé, 2011). Spécialiste des théâtres antique et contemporain, il est également rédacteur en chef de la revue Horizons/Théâtre des Presses Universitaires de Bordeaux.

Mail: Pierre.Katuszewski@u-bordeaux-montaigne.fr

Résumé : En 2017, un focus sur la création africaine subsaharienne est proposé au festival d'Avignon. Neuf spectacles sont programmés, ainsi que des conférences et des tables-rondes. Une polémique s'engage alors sur cette programmation. Cet article présente et analyse cette polémique en trois temps : tout d'abord, il revient sur la programmation et la communication officielle du festival ; ensuite, il examine les termes de cette polémique et les implications idéologiques, politiques mais surtout esthétiques, qui la sous-tendent; enfin, il pose plus glo-

Abstract : In 2017, a focus on sub-Saharan African creation is brought into attention at The Avignon Festival. The nine shows as well as discussion panels and round tables that were programmed during the festival pave the way for a new polemic to be raised. This article will present and analyze this controversy in three parts: First, it will come back to the programming and official communication of the festival, then it will examine the terms of this controversy and the ideological, political and, above all, aesthetic implications that underlie it. Finally, it will balement la question des disciplines artistiques et des étiquettes, "théâtre ", " danse ", " musique ", à une époque où de nombreux•euses chercheur euse•s s'attachent à les déconstruire et à revisiter l'histoire du théâtre qui n'est plus calquée sur l'histoire littéraire et donc des textes, mais qui s'intéresse à l'événement spectaculaire dans son entièreté.

Mots-cLés : Avignon, création africaine, disciplines artistiques, théâtre.

raise the question of artistic disciplines and labels: "theater", "dance" and "music"; at a time when many researchers are trying to deconstruct them and to revisit the history of theater, which is no longer based on literary history and therefore on texts, but which is interested in the spectacular event in its entirety.

KEYWORDS : Avignon, African creation, artistic disciplines, theater. 


\section{Retour sur la programmation du focus Afrique subsaharienne du festival d'Avignon 2017}

Depuis Qu'Olivier Py Dirige le festival D'Avignon en 2014, un focus est proposé chaque année autour d'un pays ou d'une région du monde. En 2015, c'est l'Argentine qui est à l'honneur. En 2016, c'est le Moyen Orient avec des artistes venus d'Iran, de Syrie, d'Israël, d'Égypte et du Liban. Et, en 2017, c'est au tour de l'Afrique subsaharienne avec pas moins de neuf spectacles à l'affiche. Le festival d'Avignon n'est pas en reste de polémiques, mais depuis la fameuse querelle de l'année $2005^{1}$, le calme régnait plus ou moins sous le soleil de la ville des anciens papes qui accueille ce festival depuis l'année de sa création en 1947. Si la polémique de 2017 autour du « focus Afrique subsaharienne $\gg$ fut moins médiatisée que celle de 2005, il n'en reste pas moins qu'elle fut tout aussi violente, notamment dans les journaux qui relatèrent les prises de position des un.e.s et des autres. Globalement, il est reproché à Olivier Py de ne pas avoir programmé d'auteurs ou d'autrices africain.e.s et de n'avoir proposé au public du festival que des concerts ou des spectacles chorégraphiques, ignorant toute la production dramaturgique contemporaine africaine en général et subsaharienne en particulier. Souvenons-nous qu'en 2005, Olivier Py déclarait :

Pour moi, il n'y a pas de théâtre sans récit, car il ne peut pas y avoir pensée s'il n'y a pas fable. L'idée que l'on pourrait séparer le poème du théâtre, c'est la mort du théâtre et la fin de la civilisation occidentale. Je continue à penser que le théâtre se fait à partir d'un poème; sinon, c'est du cirque ${ }^{2}$.

Cette déclaration virulente, à l'égard de toute une partie de la création occidentale contemporaine, fut sans doute provoquée par la violence de la polémique entre les tenants du théâtre à texte et ceux du théâtre dit d'image et Olivier Py, pas encore directeur du festival d'Avignon, prêchait là pour sa paroisse, lui qui monte ou bien des pièces du répertoire (Shakespeare et Eschyle notamment) ou bien ses propres pièces, longues épopées au texte foisonnant. 
Cependant, si l'on rapproche cette déclaration du sort qu'il fait à la création africaine en 2017, on est en mesure de se poser des questions. En effet, on peut avoir l'impression à l'examen du programme du festival que, pour lui, l'Afrique, c'est la danse et le chant ou, en d'autres termes, l'exotisme de peuples archaïques dont la production dramaturgique, qu'il ne peut bien entendu pas ignorer, ne serait pas assez digne d'intérêt pour être présente à l'un des festivals de théâtre les plus connus au monde. Nous ne chercherons pas plus loin les raisons qui ont poussé le directeur du festival d'Avignon à ignorer la dramaturgie subsaharienne, ce serait vain, mais ses déclarations de 2005 et la programmation du focus de 2017 permettent de soulever des questions cruciales dans le domaine des arts du spectacle; d'une part, sur la vision que certain.e.s personnes peuvent encore avoir de la création africaine et, d'autre part, sur la doxa qui a encore cours aujourd'hui, faisant du théâtre à texte l'apanage et le gage d'une culture digne de ce nom. En effet, ne pourrait-on pas retourner la polémique et affirmer que les spectacles musicaux et chorégraphiques, avec ou sans paroles d'ailleurs, sont tout aussi nobles que les autres ? Et puisque l'histoire permet souvent de légitimer le présent, rappelons une fois encore, et n'en déplaise aux défenseurs des origines et des racines de notre soi-disant théâtre, que le théâtre grec antique était tout sauf la mise en scène d'un texte ou d'un poème, qu'il n'était pas question de représenter la cité et de nourrir ou susciter la pensée des spectateurs, mais seulement de célébrer les dieux grâce à un concours musical. Seuls la performance des acteurs, les chants et les danses étaient soumis à l'appréciation d'un jury et du public. Le texte n'était qu'un matériau au même titre que la musique ou les corps dansants des acteurs.trices. Alors, on pourrait presque dire que l'Afrique subsaharienne était à l'honneur lors de ce festival et que l'absence de spectacles plus «classiques » n'est pas le signe d'un mépris de l'Afrique subsaharienne. Bien évidemment, les choses sont plus complexes que cela et il faut tenir compte des déclarations de la direction du festival, mais aussi du contexte d'accueil de ces spectacles pour mieux cerner les arguments des détracteurs de ce focus.

Trois temps viendront ponctuer notre analyse : tout d'abord, nous reviendrons sur la programmation (types de spectacle, lieux de diffusion) et la communication officielle du festival (programmes, conférences de presse); ensuite, nous examinerons les termes de cette polémique et les implications idéologiques, politiques mais aussi esthétiques, qui la sous-tendent ; enfin, nous poserons plus globalement la question des disciplines artistiques et des étiquettes, «théâtre », « danse », «musique », à une époque où, comme nous venons de le suggérer, de nombreux chercheurs s'attachent à les décon- 
struire et à revisiter l'histoire du théâtre qui n'est plus calquée sur l'histoire littéraire et donc des textes, mais qui s'intéresse à l'événement spectaculaire dans son entièreté et à des spectacles hybrides.

\section{La programmation du focus Afrique subsaharienne de la $71^{\mathrm{e}}$ édition du festival d'Avignon}

Le programme du festival est ainsi fait qu'en haut de chaque page est indiqué le genre du spectacle : «théâtre », « danse », «théâtre danse », «théâtre musique », «musique », etc., et « indiscipline » pour les spectacles hybrides mêlant plusieurs genres. Olivier Py précise bien lors de la conférence de presse de présentation du festival ${ }^{3}$ que ce sont les artistes eux-mêmes qui décident de la catégorie. Effectivement, pour les spectacles du focus, aucun n'est étiqueté «théâtre », mais « danse », « musique », 《littérature-musique », 《récit-musique » et «indiscipline ». Les auteurs de théâtre ne sont pas totalement absents, mais intégrés à un cycle de lecture en partenariat avec RFI. Il semble donc que le focus soit concentré sur d'autres types de spectacles que des pièces de théâtre à proprement parler.

Olivier Py justifie ainsi sa programmation :

[...] il se passe des choses en Afrique, parce que l'Afrique nous passionne. C'est un continent entier. Donc, il y a huit pays de l'Afrique subsaharienne qui seront présents. Ils inventent des formes, des formes « indisciplinaires 》 pour témoigner d'une réalité qui est spécifique. Et ces artistes arrivent à allier - d'une manière très originale - le combat politique et le combat artistique, l'héritage de la culture africaine auquel ils tiennent évidemment et en même temps la volonté d'une Afrique tournée vers l'avenir ${ }^{4}$.

Remarquons que le caractère « indiscipliné » qu'Olivier Py utilise pour qualifier les créations africaines est employé aussi pour des spectacles ne faisant pas partie du focus, le mélange des genres n'étant pas l'apanage de l'Afrique subsaharienne, mais faisant partie depuis plusieurs décennies déjà du paysage spectaculaire mondial! Cette déclaration semblerait aussi vouloir dire que pour parler du monde qui les entoure, les spectacles indisciplinaires seraient plus appropriés pour les Africains subsahariens que des textes de théâtre. Ce qui ne se vérifie pas bien entendu.

D'ailleurs, les textes ne sont pas absents, comme le souligne Agnès Troly, directrice de la programmation du festival : « Le texte est présent dans $80 \%$ des spectacles du focus Afrique 5 . » En effet, dans Unwanted de Dorothée Munyanega, des témoignages de femmes violées pendant le génocide au Rwanda sont énoncés ou diffusés ; le spectacle de Rokia Traore, Dream Mandé 
Djata, alterne chants et textes et on pouvait entendre des poèmes de Léopold Sédar Senghor pour Femme noire dans la Cour d'honneur du Palais des papes. Ce qui est reproché, c'est donc l'absence de pièces de théâtre, en dehors des lectures programmées en partenariat avec RFI, malgré la présence de textes dans la plupart des spectacles présentés. Nous retrouvons une fois encore les mêmes termes que lors de la polémique de 2005. Jan Fabre fut le metteur en scène/artiste associé à cette $59^{\mathrm{e}}$ édition et celui par lequel la polémique arriva. Ses créations, taxées de théâtre d'image, notamment par Olivier Py, font pourtant la part belle au texte, certaines sont même publiées. Si le conflit en 2005 tourna quasiment exclusivement autour des formes artistiques, celui de 2017 mêle les champs artistiques et idéologiques.

\section{Une polémique idéologique : le temps de la post-colonisation}

Dieudonné Niangouna, qui fut en 2013 artiste associé au festival, est le dramaturge qui lança la polémique sur le focus 2017, notamment d'un point de vue idéologique. On peut lire sur sa page Facebook:

Inviter un continent sans sa parole est inviter un mort. C'est une façon comme une autre de déclarer que l'Afrique ne parle pas, n'accouche pas d'une pensée théâtrale dans le grand rendez-vous du donner et du recevoir. Et insister en invitant cette Afrique sous cette forme muselée c'est bien pire qu'une injure. C'est inviter un mort à sa table, lui envoyer toutes les abominations à la gueule, sans se reprocher quoi que ce soit, parce que de toute évidence on sait que le mort ne parlera pas, et c'est bien la raison de cette invitation ${ }^{6}$.

Ce qui va dans le sens de ce que déclarait Olivier Py en 2005 sur l'absence de récit dans les spectacles qui furent fustigés et classés dans la catégorie du «théâtre d'image », des spectacles qui n'ont rien à voir avec ceux du focus 2017, mais le discours est le même. Finalement, on constate que les deux «opposants » ont un point de vue similaire sur la question du texte et sa fonction dans le théâtre. Nous y reviendrons dans la troisième partie de cet article. Tenons-nous en ici tout d'abord aux aspects politico-idéologiques de la polémique.

Le rapport de la France à un grand nombre de pays d'Afrique qui furent colonisés reste problématique et la polémique qui nous intéresse n'est pas exempte de ces résurgences d'un passé douloureux. Ainsi, dans un article pour Next.Libération, Ève Beauvallet pose les questions suivantes :

L'équipe du Festival d'Avignon méprise-t-elle l'Afrique et ses récits ? Ou du moins, la programmation de son "focus Afrique", dans le cadre de la $71^{\circ}$ édition 
du festival témoigne-t-elle d'une forme de paresse intellectuelle et de condescendance postcoloniale ${ }^{7}$ ?

Et en effet, quelles qu'aient été les raisons des choix de programmation, on peut constater que l'Afrique est cantonnée à l'image «traditionnelle » que peut en avoir le monde occidental, notamment un public de non-spécialistes qui ne connaîtrait pas les nombreux écrits dramaturgiques produits par ses autrices et auteurs : danses et musiques africaines sont à l'honneur - clichés et stéréotypes caractérisent certains spectacles.

Prenons en exemple Basokin, concert de musique africaine agrémenté de danses effectuées en majorité par deux femmes, parfois accompagnées d'un ou deux musiciens chanteurs, avec tous les clichés de genre que cela peut entraîner. Les conditions de la représentation et surtout de l'accueil du public sont aussi emblématiques de certains clichés : le spectacle est donné deux fois dans la même journée, une première fois à $17 \mathrm{~h}$, en plein air sous une chaleur accablante - une chaleur africaine oserait-on dire ! - et une seconde fois à $20 \mathrm{~h}$. Surtout, rien n'a été prévu pour le confort des spectateurs, pas de sièges et le simple bitume inconfortable d'une cour de collège : le public restera debout, cela va de soi, et dansera au rythme de la musique africaine. Ce qui ne serait pas un problème si les spectateurs avaient été prévenus auparavant, mais ce n'est pas le cas. Or, de nombreuses personnes âgées font partie du public, sans compter quelques VIP qui n'ont peut-être pas l'habitude d'être à même un sol franchement périlleux. Suite aux étonnements de certaines et certains, deux rangs de bancs sont installés à la va-vite par les petites-mains du festival. Quand nous arrivons pour la seconde séance, bien en avance car il est indiqué que le « placement est libre » sur le ticket, et que nous demandons si nous pouvons nous asseoir sur un de ces bancs de fortune, il nous est rétorqué que ces places sont réservées... nous allons donc prendre place par terre en attendant l'arrivée des musiciens, chanteur.euse-s et danseur.euse.s. À leur arrivée, une partie du public se lève et au fur et à mesure du concert, davantage de personnes sont prises par l'ambiance et esquissent quelques mouvements de danse, en dehors des aficionados qui dès le départ sont quasiment en transe. Le paradoxe, c'est que les personnes assises sur les bancs n’y voient rien car elles sont installées sur les côtés et un peu éloignées de la scène. Certain.e.s décident donc de se lever et de se rapprocher. Nous remarquons une personnalité très connue du monde littéraire et télévisuel français, accompagnée de son mari (africain) qui, ne tenant sans doute plus d'être assise loin du spectacle, vient s'asseoir devant la scène. Puis, vers les trois quarts 
du spectacle, elle se lève et esquisse quelques pas de danse, avant de se « lâcher $\gg$ davantage pour les derniers morceaux du concert.

Plusieurs remarques peuvent être formulées sur cet ensemble de faits : tout d'abord, mais cela pourrait se vérifier sur d'autres genres de musique, le public avignonnais n'est pas forcément habitué à assister à des concerts et moins encore dans ce genre de circonstances ; mais, surtout, nous eûmes le sentiment qu'il y avait une injonction à devoir participer à ce concert et à se laisser emporter par l'énergie débordante des performeur.euse.s. C'est de la musique et de la danse africaines, il va donc de soi que le public sera debout, en mouvement et en transe. Le groupe est reconnu, ce n'est donc pas tant la musique qui put décevoir une partie du public, mais la façon dont on nous assigna une place de voyageurs éphémères dans une cour d'école, soit un des lieux les moins accueillants de tous les lieux de spectacle du festival. Cette expérience spectatrice est un cas particulier de la programmation du focus ${ }^{8}$, mais assez emblématique des critiques qui ont pu être faites dès l'annonce de la programmation par Olivier Py en mars 2017.

Ainsi, par exemple, Qudus Onikeku, chorégraphe nigérian, voit «dans la proposition du premier [Olivier Py] une démarche au mieux en quête d'exotisme, au pire coloniale ${ }^{9} \gg$. Pour Eva Doumbia, metteuse en scène franco-ivoirienne, « il est temps de mettre fin aux relations "inégalitaires", et "de subordination", entre la France et ses anciennes colonies dans le secteur culturel $\gg$. Elle poursuit :

Dieudonné [Niangouna] est en colère, je le comprends, mais le défaut de son texte est qu'il ne décortique pas le système historico-politique qui a conduit à cette situation. Le problème de cette programmation africaine, c'est que les spectacles ont été choisis sur des sentiers balisés de la francophonie, héritage du colonialisme. Cela dénote une méconnaissance du terrain. Et il n'y a donc pas la possibilité de pluralité dont parle Rokia Traoré, qui permettrait de faire aussi entendre du théâtre textuel d'Afrique ${ }^{10}$.

Citons Dieudonné Niangouna qui fustige donc violemment cette programmation avec des dramaturges relégués à la radio :

C'est bien de ça qu'il s'agit dans cette programmation de la soixante et onzième édition du Festival d'Avignon, soit cinquante-sept ans après la décolonisation des espaces francophones africains. Cinquante-sept ans après, c'est énorme! On ne peut quand-même pas continuer à jouer avec un continent. L'impression que certains peuvent tout dire, tout faire, et faire-faire aux créateurs issus du continent africain sans qu'aucun raisonnement sensé au préalable ne puisse nourrir la réflexion. Les créateurs de théâtre issus du continent africain ce sont des pas 
aboutis pour eux, des dégénérés peut-être... Sinon comment peut-on accepter de retirer la parole dans un corps? Mais enfin, sommes-nous revenus à l'époque de Hérodote où l'on disait que le noir n'est que bruit, son et tam-tam? Dans un festival de théâtre en plus ${ }^{11}$ ?

Qu'il s'agisse d'une «maladresse » de la part de la direction du festival d'Avignon, comme le pensent certains metteurs en scène venus à la rescousse d'Olivier Py, sans nul doute, mais à l'heure où les débats sont vifs sur la place des minorités visibles sur les scènes de théâtre, ce focus sans véritables textes de théâtre ne pouvait que mettre le feu aux poudres.

Au-delà de cette polémique autour des résidus de la colonisation et d'une vision perçue comme dominante et méprisante de l'Afrique, le conflit porte donc sur les genres spectaculaires. Abordons les termes de cet antagonisme afin de voir que, sous cet aspect, les deux parties qui s'opposent sont finalement proches d'un même paradigme esthétique que nous pouvons qualifier d'aristotélicien et qui ignore, nous l'avons rapidement évoqué plus haut, les récentes avancées dans le domaine de l'histoire du théâtre. Alors, comme le demande Ève Beauvallet : «La présumée "domination post-coloniale” dont aurait fait preuve la direction en n'invitant aucun artiste africain étiqueté "théâtre ". Vrai débat idéologique ou vieille querelle esthétique ${ }^{12}$ ? »

\section{Une polémique culturelle : Aristote encore et toujours}

L'absence de «théâtre » serait donc un acte de mépris envers un continent dont la production dramaturgique est florissante. Cependant, si l'on abandonne un moment ces querelles idéologiques, car malgré l'importance qu'elles peuvent avoir, il est impossible de les résoudre, on pourrait renverser les choses et voir dans ce focus plus musical et chorégraphique que théâtral l'inscription d'une partie de la production africaine dans l'ensemble des spectacles contemporains pour lesquels les créateurs usent de plusieurs médiums et plus seulement du texte. En effet, en dehors de Basokin et du récital de Rokia Traoré, les autres spectacles programmés du focus sont des créations hybrides, indisciplinées comme les qualifie Olivier Py, très ressemblants à bien d'autres spectacles contemporains. Certes, à la lecture du programme, une majorité de spectacles sont étiquetés 《 théâtre » ou 《danse », et quasiment seuls les spectacles du focus sont «indisciplinés », mais dans le paysage théâtral, les spectacles du focus sont tout à fait dans l'air du temps.

C'est donc bien à une « querelle esthétique » à laquelle on assiste également. Et on pourrait renvoyer dos à dos la direction du festival et ses détrac- 
teurs, Dieudonné Niangouna au premier chef. C'est dans ce sens que l'on peut lire les déclarations de Jan Gossens au journal Libération. Il se dit

à la fois respectueux du travail de Dieudonné Niangouna, à la fois très perturbé par cette vision extrêmement réductrice de la création contemporaine africaine. Sa vision de l'art dramatique comme lieu exclusif du texte littéraire (une tradition plutôt française) n'est pas du tout centrale en Afrique où les traditions font plus de place au mouvement, au chant, aux traditions orales bien sûr. Le texte littéraire sur les plateaux n'est venu qu'avec les projets coloniaux [...]. Au vu de cette réalité, le focus me semble légitime. Est-ce qu'il est complet? Je ne pense pas que ce soit l'idée. Il est complémentaire avec ce qui a déjà pu être présenté au Festival d'Avignon en 2013, par exemple, lorsque Dieudonné Niangouna était artiste associé. Mais dire que des artistes comme Serge Aimé Coulibaly ou Dorothée Munyaneza ne sont pas des représentants légitimes du théâtre africain contemporain, c'est comme dire qu'Alain Platel ou Jan Lauwers ne sont pas des représentants légitimes du théâtre contemporain flamand ${ }^{13}$.

Nous retrouvons ici la même sorte d'opposition qui émergea en 2005 entre les défenseurs du théâtre à texte et ceux du théâtre d'image que l'on peut rapprocher des spectacles dits «indisciplinés». Comme l'écrit Florence Dupont dans l'introduction de son ouvrage sur Aristote :

Or, les arguments des uns et des autres, en ce mois de juillet 2005, montrent $q u$ 'une révolution intellectuelle reste à faire qui change les termes $d u$ débat, afin qu'on sorte, par exemple, du conflit qui n'a pas lieu d'être, entre théatre à texte et théatre du corps.

Elle poursuit : « En fait, le débat tourna autour de la défense du théâtre à texte, qui serait seul du vrai théâtre, et du mélange des arts qui évacue ou marginalise volontiers le texte. » Cette façon de considérer le texte vient de ce que l'histoire du théâtre a longtemps été, et est encore trop souvent, calquée sur l'histoire littéraire - suivant en cela Aristote pour lequel « le reste - jeu, spectacle, masque, etc. - vient après le "poème" ». Elle renvoie alors dos à dos Jan Fabre et Olivier Py, le premier représentant le théâtre d'image et l'autre le théâtre à texte, tous les deux étant aussi aristotéliciens l'un que l'autre à la lecture des intentions de leurs spectacles : «Le nietzschéen Jan Fabre, fasciné par l'illusion des origines, est tout aussi pris dans le piège aristotélicien qu'Olivier $\mathrm{Py}^{14}$. »

De la même façon, les défenseurs à la fois $d u$ « théâtre » africain mais aussi des spectacles indisciplinés du focus 2017 ont des arguments tout à fait aristotéliciens. Par exemple, Jean-Louis Sagot-Duvauroux rappelle que pour lui $\ll$ la danse parle $»$ : 
Dans les civilisations d'Afrique de l'Ouest, la danse fait partie de la culture générale. Elle joue un rôle important de liberté d'expression: dire ce que les corps, habituellement contenus par les règles de la civilité, ne peuvent exprimer dans la vie courante; le dire par des mises en forme qui affirment la dignité humaine jusque dans l'expression de la violence ou de l'érotisme. Ce qui se construit au sud du Sahara sous le label occidental de danse contemporaine est de moins en moins coupé de cette inspiration et vit aujourd'hui, en Afrique, un tournant qui mérite l'attention. Beaucoup de pièces ont une trame narrative explicite et usent du texte ${ }^{15}$.

Et il a bien rappelé, notamment par Agnès Troly, que le spectacle de Rokia Traoré est construit autour d'un récit. Surtout, une course pour savoir à qui reviendrait, au cours de l'histoire, l'inclusion du texte dans les spectacles africains est déclarée par journaux et blogs interposés. Pour Serge Aimé Coulibaly, « la séparation de la parole et du corps n'est absolument pas burkinabée. Elle est arrivée avec la pratique occidentale du théâtre ${ }^{16} \gg$. Quant à Jan Goossens, il indique, nous l'avons vu, que « le texte littéraire sur les plateaux n'est venu qu'avec les projets coloniaux $\gg$. Ce à quoi Jean-Louis SagotDuvauroux lui répond que

c'est un raccourci spécieux. Un exemple: dans les années 80 , des auteurs, metteurs en scène et comédiens bamakois font monter la longue histoire du kotèba (farces burlesques de satire sociale) sur la scène du Palais de la Culture de Bamako. [... ] le public malien n'a aucun doute sur l'ascendance endogène de cette innovation, sur la vérité de «l'esprit kotèba » qui en est l'âme. Ce renouveau théâtral est jusqu'à présent explicitement associé par l'opinion au mouvement de révolte qui abattra en 1991 la dictature de Moussa Traoré. Projet colonial sous prétexte que cet «esprit » se joue sur une scène et dans une grande ville ? L'opinion saugrenue de Jan Goossens est intenable ${ }^{17}$.

On le voit bien, la présence du texte et du discours qui l'accompagne légitime la valeur culturelle des créations théâtrales. Ce qui n'est pas sans rappeler, une fois encore, les critiques qui furent adressées à Jan Fabre en 2005 sur la supposée absence de sens, comme par Rosita Boisseau dans sa critique pour le journal Le Monde : «Très attendue, L'Histoire des larmes de l'artiste flamand a ouvert le $59^{\mathrm{e}}$ Festival d'Avignon, vendredi 8 juillet. Un métissage de théâtre et de danse dont la beauté plastique ne masque pas le déficit de sens ${ }^{18}$. $\gg$ Si les arguments des uns et des autres sont légitimes - et il ne s'agit pas ici de trancher -, nous pouvons constater qu'ils se situent dans un paradigme aristotélicien qui semble indépassable. La présence du texte, et du sens qu'il sous-tend, justifierait la valeur d'un spectacle, le texte soit entendu comme texte dramatique, soit que l'on pourrait qualifier de « corporel $\gg$. 
Dans un cas comme dans l'autre, il s'agit toujours de dire quelque chose au public, de représenter d'une façon ou d'une autre le monde extérieur au théâtre et de tenir un discours sur ce dernier, en oubliant que le théâtre n'a pas toujours eu cette fonction, entre autres le théâtre grec antique dont on dit encore trop souvent qu'il est l'origine du théâtre occidental.

\section{Abolir les catégories, retrouver le spectacle}

Revenons aux spectacles de ce focus et à la façon dont ils sont décrits dans le programme du festival d'Avignon. Si, pour deux spectacles, la description a de quoi provoquer la polémique par les clichés et stéréotypes qu'ils charrient $^{19}$, pour les autres, l'aspect politique et parfois presque militant qu'ils proposent aurait dû satisfaire les détracteurs de cette programmation. Mais non, si le théâtre n'est pas reconnu pour sa littérature, c'est le déni de tout un continent. C'est certes compréhensible en partie puisque l'Afrique regorge de dramaturges et qu'il n'y a aucune raison à ce qu'ils n'aient pas été invités en 2017, mais cela démontre aussi l'importance donnée au texte.

Deux querelles sont donc apparues au cours de notre analyse. La première est idéologique, et elle n'est pas seulement réservée au festival d'Avignon 2017, mais s'inscrit au sein des revendications et questionnements contemporains sur la place des minorités dites « visibles » ou de la diversité sur les scènes de théâtre, notamment en France, pays marqué par son passé colonial et postcolonial. Si les spectacles du focus ne sont pas le fait de créateur.trices français.e.s ou vivants en France, la polémique ne pouvait qu'émerger à une période, la nôtre, révélant une grande sensibilité sur cette problématique. Dans le numéro récent de la revue Alternatives théâtrales consacré à la question, Sylvie Chalaye déclare que

prendre conscience que nous devons sortir de la postcolonie, c'est ne plus enfermer les imaginations de l'altérité dans les marges exotiques de la création contemporaine, afin que leur inventivité soit partie prenante de la culture nationale et stimule au contraire sa vitalité de l'intérieur ${ }^{20}$.

C'est bien ce que tenta de faire Olivier Py, mais il « oublia » les dramaturges subsahariens.

Ce qui entraîne une seconde polémique sur les genres spectaculaires et leur hiérarchie. L'histoire des spectacles a tellement sublimé l'idée de la suprématie du texte au théâtre que toutes les autres sortes de spectacles sont « naturellement » pourrait-on dire, moins nobles - même s'ils comportent du texte et encore plus quand ils proviennent de pays ou continents comme 
l'Afrique, reléguée alors aux stéréotypes encore très ancrés dans les mentalités à notre époque.

Pour conclure, reprenons les questions que Florence Dupont pose en introduction de son ouvrage L'Orateur sans visage à propos du théâtre romain :

Quand se souviendra-t-on de Rome? Quand en finira-t-on avec l'oubli volontaire des scènes romaines? Quand acceptera-t-on qu'un théâtre des origines, qui fut un théâtre du jeu et rien qu'un théâtre du jeu, fasse irruption sur notre horizon intellectuel et bouscule bien des fausses évidences, bien des naïvetés historiques? La modernité prendrait alors un autre sens qui ne serait plus le sens de l'histoire ${ }^{21}$.

Briser un certain continuum historique et se souvenir que le théâtre ne mit pas toujours le texte au centre de ses pratiques permet de ne plus établir de jugements de valeur, ni entre les différents genres spectaculaires, ni entre les différentes civilisations. Alors si le théâtre africain est bien évidemment incomparable avec le théâtre romain, puisqu'il comporte tous les genres de spectacles, le considérer pour ce qu'il est, dans la multitude de ses propositions, et plus à l'aune de critères aristotéliciens et occidentaux, permettrait d'éviter de telles polémiques.

Plus généralement, il serait peut-être temps d'abolir les genres et les classifications et de ne plus faire de focus sur une aire géographique en particulier, mais de mêler la création africaine aux autres sans la mettre plus particulièrement en lumière, comme cela se fait parfois. Ne pourrions-nous pas, comme le préconise Maurizio Bettini, nous ériger « contre les racines » et la tradition ? Ce qui éviterait bien des malentendus et des controverses ; car, « malgré les énormes différences culturelles entre passé et présent, on soutient avec toujours plus d'insistance que ce que nous avons été - ou plutôt, ce que nous aurions été, selon certains - est la seule chose capable de nous dire qui nous sommes aujourd'hui. Comme si la tradition avait le pouvoir de sculpter dans le marbre les contours de notre identité collective $\mathrm{e}^{22} \gg$ et $\ll$ la tradition, en effet, n'est pas une chose qui pousse de la terre, qui se mange ou qui se respire, ni une chose qui descend vers nous de certaines hauteurs : c'est d'abord une chose qui se construit et qui s'apprend ${ }^{23} \gg$.

\section{Notes}

1. Cf. Georges Banu, Bruno Tackels (dir.), Le Cas Avignon 2005: regards critiques, Montpellier, L'Entretemps, 2005 ; Carole Talon-Hugon, Le Conflit des héritages. Avignon 2005, Arles, Actes Sud-Papiers, 2017 [2006] ; Régis Debray, Sur le pont d'Avignon, Paris, Flammarion, 2005. 
2. Cité dans Fabienne Darge, «À la recherche de nouvelles formes de récits », Le Monde, 4 juillet 2005.

3. URL : https://www.theatre-video.net/video/Conference-de-presse-du-71e-Festivald-Avignon-presentation-par-Olivier-Py. [Consulté le 7 février 2018.]

4. Cité dans Siegfried Forster, "L'Afrique sera au cœur du Festival d'Avignon 2017 ", RFI, 23 mars 2017. URL : http://www.rfi.fr/culture/20170323-afrique-coeur-festivalavignon-2017-olivier-py. [Consulté le 28 octobre 2017].

5. Citée dans Clarisse Fabre, Séverine Kodjo-Grandvaux, «Festival d'Avignon : la scène africaine veut "décoloniser" les regards ", Le Monde, 3 juillet 2017.

6. Le texte est reproduit intégralement sur le site sceneweb.fr. URL : https://www. sceneweb.fr/le-coup-de-colere-de-dieudonne-niangouna-sur-la-programmation-du-festival-davignon-2017/ [Consulté le 7 février 2018].

7. Ève Beauvallet, "Le focus "Afrique" du Festival d'Avignon divise ", Next Libération, 27 mars 2017. URL : http://next.liberation.fr/culture-next/2017/03/27/le-focusafrique-du-festival-d-avignon-divise_1558703 [Consulté le 28 octobre 2017].

8. Ce fut très différent lors du spectacle de Rokia Traoré, déjà star en France, et donc invitée à se produire dans la magnifique cour du musée Calvet.

9. Cité dans Clarisse Fabre, Séverine Kodjo-Grandvaux, "Festival d'Avignon : la scène africaine veut "décoloniser" les regards ", op. cit.

10. Citée dans ibid.

11. URL : https://www.sceneweb.fr/le-coup-de-colere-de-dieudonne-niangouna-sur-laprogrammation-du-festival-davignon-2017 [Consulté le 7 février 2018].

12. Ève Beauvallet, «Le focus «Afrique » du Festival d'Avignon divise », op. cit.

13. Ibid.

14. Florence Dupont, Aristote ou le vampire du théâtre occidental, Paris, Flammarion, 2007, p. 17-21.

15. Jean-Louis Sagot-Duvauroux, "Focus Afrique" en Avignon. Une polémique utile », URL : https://jlsagotduvauroux.wordpress.com/2017/03/29/focus-afrique-en-avignon-une-polemique-utile/[consulté le 7 février 2018].

16. Cité dans Clarisse Fabre, Séverine Kodjo-Grandvaux, « Festival d'Avignon : la scène africaine veut « décoloniser» les regards ", op. cit.

17. Jean-Louis Sagot-Duvauroux, « "Focus Afrique » en Avignon. Une polémique utile », op. cit.

18. Rosita Boisseau, "Le corps selon Jan Fabre laisse Avignon perplexe ", Le Monde, 9 juillet 2005.

19. Ainsi, Basokin est propose une musique "enracinée très loin dans une culture traditionnelle éloignée des villes modernes [...]. Le spectacle raconte une Afrique inattendue, à la fois mystérieuse et hédoniste, millénaire et actuelle », programme du festival d'Avignon 2017, p. 32 ; The Last King of Kakfontein de Boyzie Cekwana est « à la fois conte sauvage et tragédie shakespearienne ", ibid., p. 36.

20. Sylvie Chalaye, "Peau et incarnation, des impensés politiques de la scène contemporaine ", dans Quelle diversité culturelle sur les scènes européennes? Alternatives théâtrales, $\mathrm{n}^{\circ} 133$, novembre 2017, p. 27-35, l'extrait cité se trouve p. 32.

21. Florence Dupont, L'Orateur sans visage. Essai sur l'acteur romain et son masque, Paris, PUF, 2000, p. 1. 
Retour sur la programmation du focus Afrique subsaharienne du festival d'Avignon 2017

22. Maurizio Bettini, Contre les racines, trad. de l'italien par Pierre Vesperini, Paris, Flammarion, 2017, p. 19. L'auteur souligne.

23. Ibid., p. 53. 